\title{
DOSAGGIO DI ANTICORPI ANTI PEPTIDE CICLICO CITRULLINATO IN PAZIENTI CON ARTRITE REUMATOIDE
}

Garlaschi M.C.*, Paina S. ${ }^{\circ}$, Monti M.A. ${ }^{\circ}$, Calmi S.*, Bonamore R.*, Restelli A.*, Maiavacca R.^, Torresani E*.

*U.O. Microbiologia, IRCCS Fondazione Policlinico,

Mangiagalli e Regina Elena

ソU.O. di Medicina Interna, IRCCS

Fondazione Policlinico, Mangiagalli e Regina Elena

^U.O. di Patologia Clinica, IRCCS Fondazione Policlinico,

Mangiagalli e Regina Elena.

Introduzione. L'Artrite reumatoide (AR) è una malattia autoimmune sistemica, caratterizzata dall'infiammazione delle membrane sinoviali. La diagnosi di Artrite Reumatoide è principalmente clinica, radiologica e immunologica. Il test immunologico più frequentemente utilizzato è la ricerca del Fattore Reumatoide (FR) (American College of Rheumatology). Il FR è ritrovato nel $60-80 \%$ dei pazienti con $\mathrm{AR}$, ma altrettanto presente nel $30 \%$ dei pazienti, con altre patologie autoimmunitarie o con infezioni croniche. Nel 1998, Schellekens mette a punto un test ELISA basato sul peptide ciclico citrullinato $(\mathrm{CCP})$. La buona sensibilità ma soprattutto l'elevata specificità ( $96 \%$ ) riportate in letteratura, ci spingono a valutare un test ELISA basato sulla presenza dell'anticorpo ciclico citrullinato (CCP).

Lo scopo dello studio è quello di pervenire ad una diagnosi precoce nei casi sospetti per AR ed inoltre di valutare la fase evolutiva della malattia stessa correlando la titolazione con l'entità della fase erosiva osteoarticolare della AR.

Pazienti e metodi. Nell'anno 2005 abbiamo arruolato 38 pazienti, di cui 10 con diagnosi di AR e 28 con altre patologie autoimmunitarie (gruppo controllo).

I pazienti sono stati sottoposti ad esami strumentali, comprese radiografie dello scheletro, e ad esami ematochimici . Sono stati dosati gli anticorpi anti CCP, con una metodica ELISA (Axis-Shield, Ditta Bouty) e il FR con una metodica immunoturbidimetrica quantitativa (Ditta Roche)

Risultati. Dei 28 campioni provenienti dai pazienti controllo sono risultati 27 negativi, in un solo campione si è ottenuto un risultato dubbio, (Valore medio pari a $2.0 \mathrm{U} / \mathrm{ml}$ ( cut off $5 \mathrm{U} / \mathrm{ml}$ ).

Dei 10 campioni ematici provenienti da pazienti con diagnosi di AR, 6 campioni sono risultati francamente positivi con valore medio pari a $119.3 \mathrm{U} / \mathrm{ml}$ (cut off $5 \mathrm{U} / \mathrm{ml}$ ). Gli altri 4 campioni sono risultati negativi; su questi ultimi si è osser-

vata peraltro una buona risposta alla terapia in corso. Conclusione. Il test discrimina in modo netto la popolazione controllo da quella con AR, ed inoltre supporta il clinico in modo efficace nella gestione della terapia di fondo e di conseguenza sulla evoluzione della malattia, soprattutto dal punto di vista del danno articolare. 